
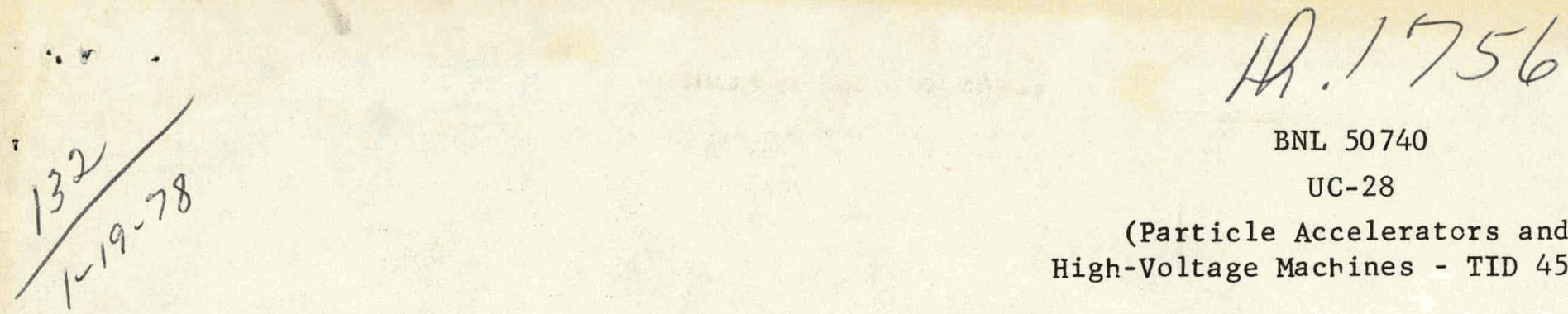

BNL 50740

UC-28

(Particle Accelerators and

High-Voltage Machines - TID 4500)

\title{
COMMENTS ON MUON TRAPPING
}

A.J. Stevens and A.M. Thorndike

June 16, 1977
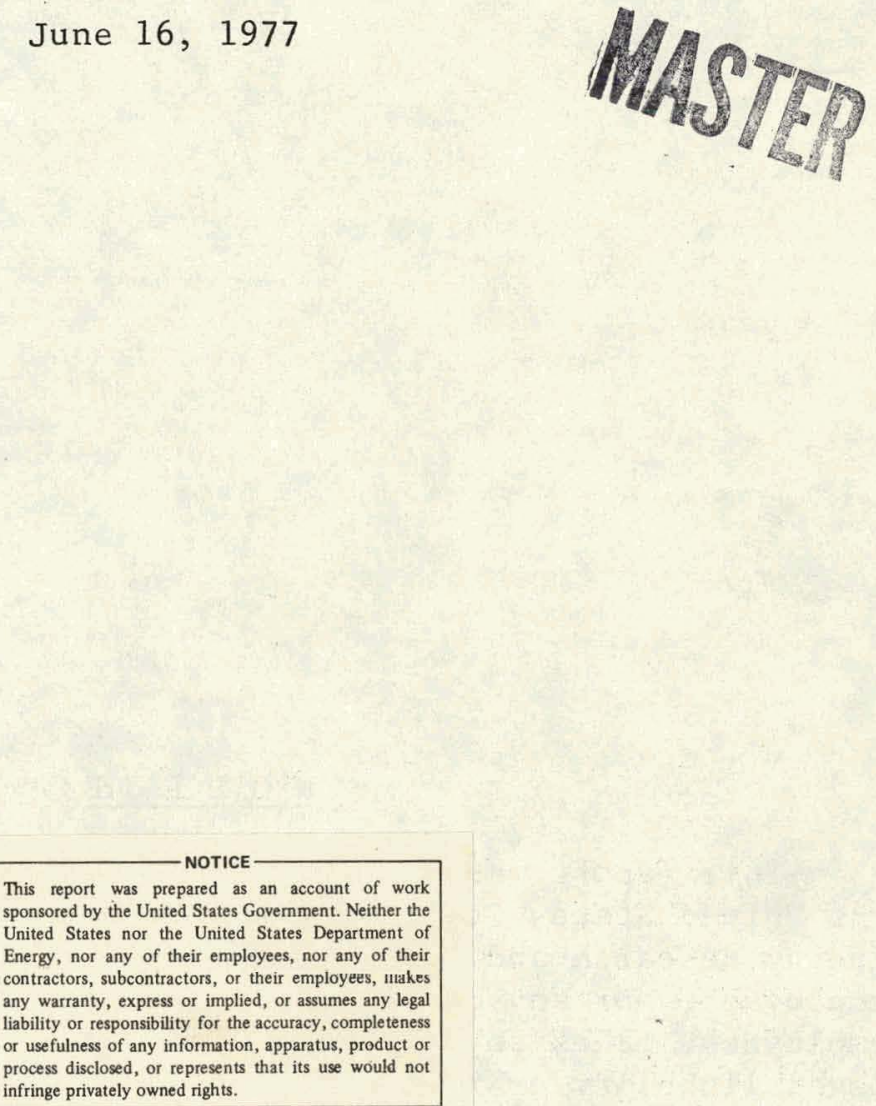

ACCELERATOR DEPARTMENT

B R O O K H A V E N N A T I O N A L L A B O R A T O R Y A S S OC I A T E D U N I V E R S I T I E S, I N C. U P T O N, N E W Y O R K 11973 


\section{DISCLAIMER}

This report was prepared as an account of work sponsored by an agency of the United States Government. Neither the United States Government nor any agency Thereof, nor any of their employees, makes any warranty, express or implied, or assumes any legal liability or responsibility for the accuracy, completeness, or usefulness of any information, apparatus, product, or process disclosed, or represents that its use would not infringe privately owned rights. Reference herein to any specific commercial product, process, or service by trade name, trademark, manufacturer, or otherwise does not necessarily constitute or imply its endorsement, recommendation, or favoring by the United States Government or any agency thereof. The views and opinions of authors expressed herein do not necessarily state or reflect those of the United States Government or any agency thereof. 


\section{DISCLAIMER}

Portions of this document may be illegible in electronic image products. Images are produced from the best available original document. 


\section{NOT I C E}

This report was prepared as an account of work sponsored by the United States Government. Neither the U.S. nor the U.S. Energy Research and Development Administration, nor any of their employees, nor any of their contractors, subcnntractors, or their employees, makes any warranty, express or implied, or assumes any legal liability or responsibility ful llie accuracy, complereness or usefulness of any information, apparatus, product or process disclosed, or represents that its use would not infringe privately owned rights.

PRINTED IN THE UNITED STATES OF AMERICA Available from

National Technical Information Service

U.S. Department of Commerce 5285 Port Royal Road Springfield, VA 22161

Price: Printed Copy $\$ 4.00$; Microfiche $\$ 3.00$ 


\begin{abstract}
Muons that result from the collisions of protons in the ISABELLE beams with other nucleons introduce important background or signal for experiments. Their ability to pass through the conductors and steel of ISABELLE magnets results in complicated trajectories. Some oscillate about quasistable orbits and are called "trapped".

A number of typical trajectories have been plotted, and flux estimates have been made with a Monte Carlo program, HALO. Trapping tends to reduce shielding requirements somewhat, but the effect does not appear to be a very large one from these initial investigations.

More aspects need to be studied, and HALO appears to be a useful tool fur doing so.
\end{abstract}




\section{Introduction}

When a proton in one of the ISABELLE beams collides with any other nucleon, secondary pions and kaons may be produced that decay into muons. In addition, muons are produced directly with a cross section about $10^{-4}$ that of pions. This occurs for beam-beam collisions, beam-wall collisions, and for any beam loss at scrapers or other points where protons with the largest oscillations hit vacuum walls or other solid material. Energetic muons penetrate large amounts of material. For this reason muons have a special interest with respect to shielding requirements, anticipated backgrounds for some experiments, and may possibly be a useful tool for monitoring beam behavior.

In discussions of ISABELLE the need for muon shielding was recognized some time ago, and the discussion of shielding requirement estimates in ISA 76-11 led to a very thick muon shield. The thickness required is determined primarily by high energy muons at small angles to the proton beam. The procedure used in ISA 76-11 was a very simple one in which the muons were considered to emerge tangentially with energies derived from Wang's empirical formula for secondary pion spectra. This ignores deflection by the magnetic fields of the ISABELLE magnets.

During the 1976 ISABELLE Workshops a review of shielding estimates was made ${ }^{2}$. It was pointed out that neglect of magnetic deflection was an approximation that was nint likely to be a good one. The deflections caused by magnetic fields can be substantial, and an early study by Keefe and Scolnick ${ }^{3}$, of orbits in the lattice of the $200 \mathrm{GeV}$ accelerator carried out at Berkeley, had indicated that positive muons were very frequently "trapped" by the combination of the guide field and the return field in the yoke of the magnets ${ }^{3}$.

1. A.J. Stevens and A.M Thorndike, "Estimating ISABELLE Shielding Requirements", ISA 76-11, April 5, 1976 .

2. T. Ludlam; V. Kistiakowsky', and T.E. Toohig, Proc. 1976 ISABELLE Workshops, p. 144, BNL 50611 .

3. D. Keefe and M. Scolnick, "Trapping of $\mu$ - mesons in Magnet Structures", UCii) 10143 (Sept. 14, 1965). 
The Workshop report indicated " that the trapping of high energy $\mu$ mesons by the ring magnets may substantially reduce the required amount of shielding. To the extent that such trapping occurs, it may tend to enhance the muon background in experimental halls".

We have been investigating this question and feel that our understanding of it has improved sufficiently that a report is worth while at the present time. We confirm the existence of trapped orhits in the ISABELLE lattice, similar to those reported by Keefe and Scolnick. The orbits are quite complicated, however, and we feel that it is not easy to make broad generalizations about them.

This report will give a highly simplified example of "trapped" orbits in Section 2. Then section 3 will give illustrative orbits in two dimensions and Section 4 will extend this to some graphical representations of orbits in three dimensions.. To provide some synthesis of the varied possibilities we have used the Monte Carlo program, HALO, prepared at CERN for the. study of muon distributions around secondary beams ${ }^{4}$. Some of the muon flux distributions generated by HALO are given in Ecction.5. Section 6 gives some qualitative conclusions with reference to the muon shielding provisions of the 1977 ISABELLE Proposa ${ }^{5}$.

\section{A Simplified Trapping Example}

In Fig. 1 a ring-shaped reginn, $B$, is chown in which we lmagine an upward magnetic field, while the magnetic field is downward in. Lhe adjacent regions, $A$ and $C$. There is an obvinus resomblance to the uedian $\ddot{p}$ lane of a synchrotron, with $A$ and $C$ being the return yoke of the magnet. The example considers two dimensions only. Assume. the field in $A$ and $C$ is half $a$ a big as $B$. The dotted line is the proton beam orbit.

4. Ch. Iselin, "HALO, A Computer Program to Compute Muon Halo", CERN 74-17, Laboratory II, Experimental Areas Group, 29 August 1974.

5. "ISABELLE, A Proton-Proton Colliding Beam Facility", BNL 50648, Apri1 1977. 
A

PROTON BEAM

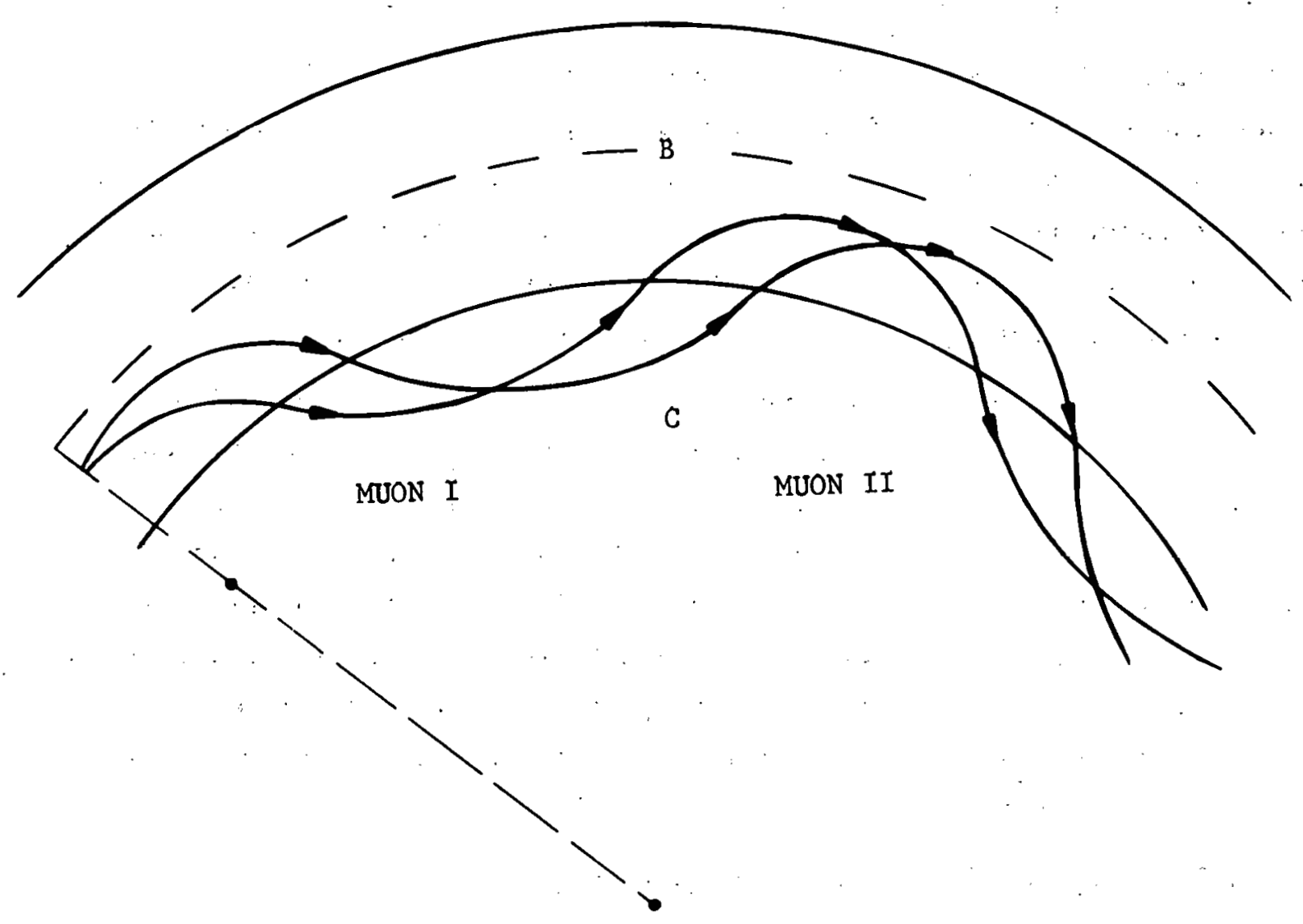

\begin{tabular}{|c|c|c|c|}
\hline & 1 & 1 & 1 \\
\hline P & $\begin{array}{l}\text { MUON } \\
\text { RADIUS } \\
\text { IN B }\end{array}$ & $\begin{array}{l}\text { MUON } \\
\text { RADIUS } \\
\text { IN C }\end{array}$ & $\begin{array}{l}\text { PROTON } \\
\text { RADIUS }\end{array}$ \\
\hline
\end{tabular}

Fig. 1. Simplified trapped trajectories, magnetic field in regions $A$ and C opposite to B 
Take a muon whose momentum is $1 / 4$ that of the protons, or $50 \mathrm{GeV}$. It can penetrate a lot of iron with little scattering or energy loss, so ignore these effects. Its trajectory soon enters region $C$, as shown, and then bends back the other way. So long as regions $B$ and $C$ extend far enough this goes on forever, as in the trajectories shown.

of course this trapping is a two-dimensional effect which does not provide focussing in three dimensions. Only the protons do that. Keefe and Scolnick showed, however, that the muons do not move out of the median plane very fast, and argued that two-dimensional trajectories give a useful understanding of the real three-dimensional behavior. Such trajectories are plotted in section 3 for geometry like that of ISABELLE.

In Fig. 1 the field is imagined to reverse abruptly in going from region $B$ to region $C$, which is unphysical. There has to be a continuous function, which looks like Fig. 2 for ISABELLE dipoles. At the point marked with the arrow the field has the right value for a $50 \mathrm{GeV}$ muon to have the same curvature as the protons. This can be thought of as a stable muon orbit. Actual muons have trajectories that oscillate about it. Vertical motion, scattering, energy loss, and different field shapes in quadrupole magnets cause the oscillations to tend to blow up rather quickly, and muon trajectories have a lot of variations.

\section{Illustrative Two-Dimensiona1 Trajectories}

The trajectories we are inţerested in penetrate the cnils and steel cores of the ISABELLE magnets. We have found no general description for such behavior. Consequently a computer program was prepared to track the muons through fields that approximate the median-plane distribution in ISABELLE. It includes energy loss, but scattering is neglected. Scattering is considerably smaller than magnetic deflection for the fields involved, so this is a good approximation. Trajectories were plotted for a number of source positions and a number of momenta.

From the shielding point of view muons of very high momentum are not important because there are so few of them they are no problem. Muons of 


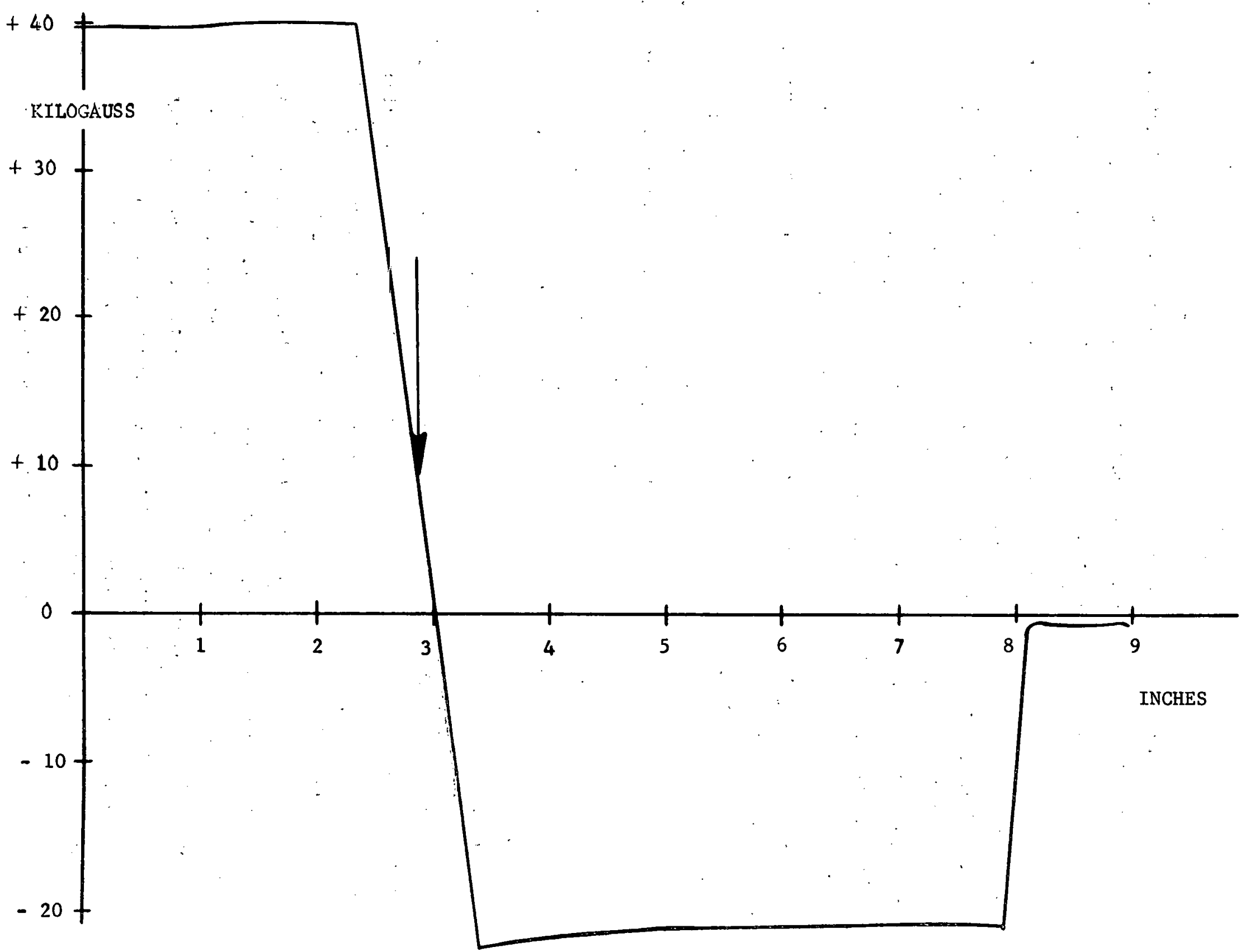

Fig. 2. Calculated magnetic field on median plane (from George Parzen) 80 ribbons, 6 blocks, BLG $-4.15,40 \mathrm{kG}$. 
very low momentum are no problem because a thin shield is enough for them. The region of interest proves to be about $50 \mathrm{GeV} / \mathrm{c}$. We show trajectories for $60 \mathrm{GeV} / \mathrm{c}$ since higher momenta are more favorable for trapping.

In Fig. 3 we show trajectories from a point $6 \mathrm{~cm}$ from the beam at the intersection point, where the vacuum wall might be located. There are three different kinds of trajectories, according to the part of the first quadrupole doublct $(Q 1, Q 2)$ tilal the muons hit.

1) Within the quadrupolc aperture: These muons are focussed to a point a short distance downstream of $\mathrm{Q} 2$ and emerge from this image with increased angular divergence. (This is also true in thrce dimensions.) There is a small angular range that hits Q3 and Q4 in positions favorable for trapping in the subsequent lattice. One such trajectory is shown.

2) Passing through coils of Q1 and Q2: The large fields and gradients in and near the magnet coils produce very strong deflections. Cross-overs resembling foci exist in the quadrupole magnets. The effect is to increase angular divergence in a rather random way, much like scattering.

3) Passing through the core of $Q 1$ and $Q 2$ : The deflections are opposite in sense and roughly equal so these trajectories are not greatly altered and appear to diverge from the original source. The same, of course, is true of trajectories that pass outside Q1 and Q2 altogether.

In Fig. 4 we show similar trajectories for a source at a quadrupole in the regular lattice. Here the wall is $4 \mathrm{~cm}$ from the central proton orbit and is taken on the side closer to the stable muon orbit on the inner sidc of the magnet aperture. This is a position favorable to muon trapping. The figure shows 21 trajectories, 4 of which are trapper sufficiently to emerge at the downstream face of the 10th magnet alument. MOsE of the trajectories are lost to the outside of the magnet structure after one or two complete oscillations about the stable orbit. They pass through considerable steel in the process, but they leave at a steep angle. It is not obvious whether the overall effects of the magnetic fields are helpful or harmful from a shielding viewpoint.

A number of trajectories have been plotted similar to those in Fig. 4. 


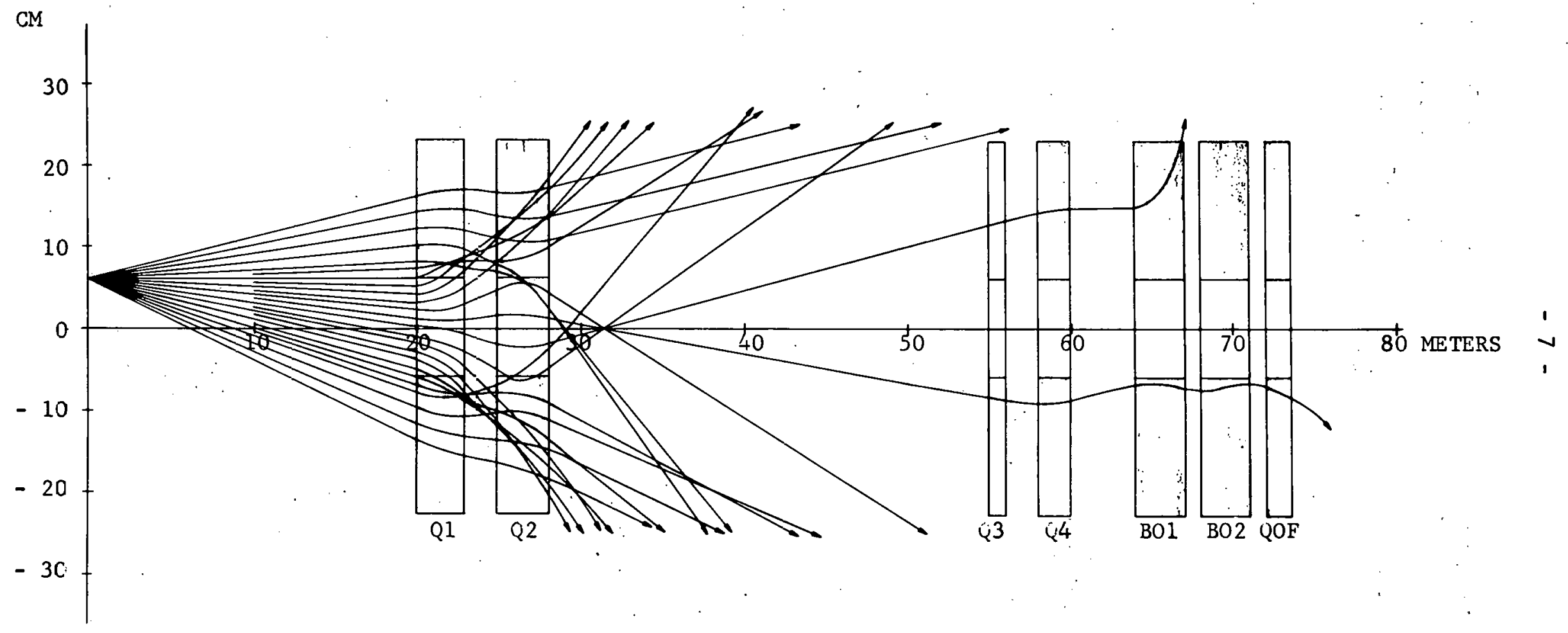

Fig. 3. Trajectories for $60 \mathrm{GeV} / \mathrm{c} \mathrm{h}^{+}$ originating from a source at vacuum chamber wall at the crossing point for the two-dimensional case. Distances along beam in meters, and perpendicular in centimeters. One trajectory is "trapped" 73 meters downstream. 
$\mathrm{X}$ in $\mathrm{cm}$

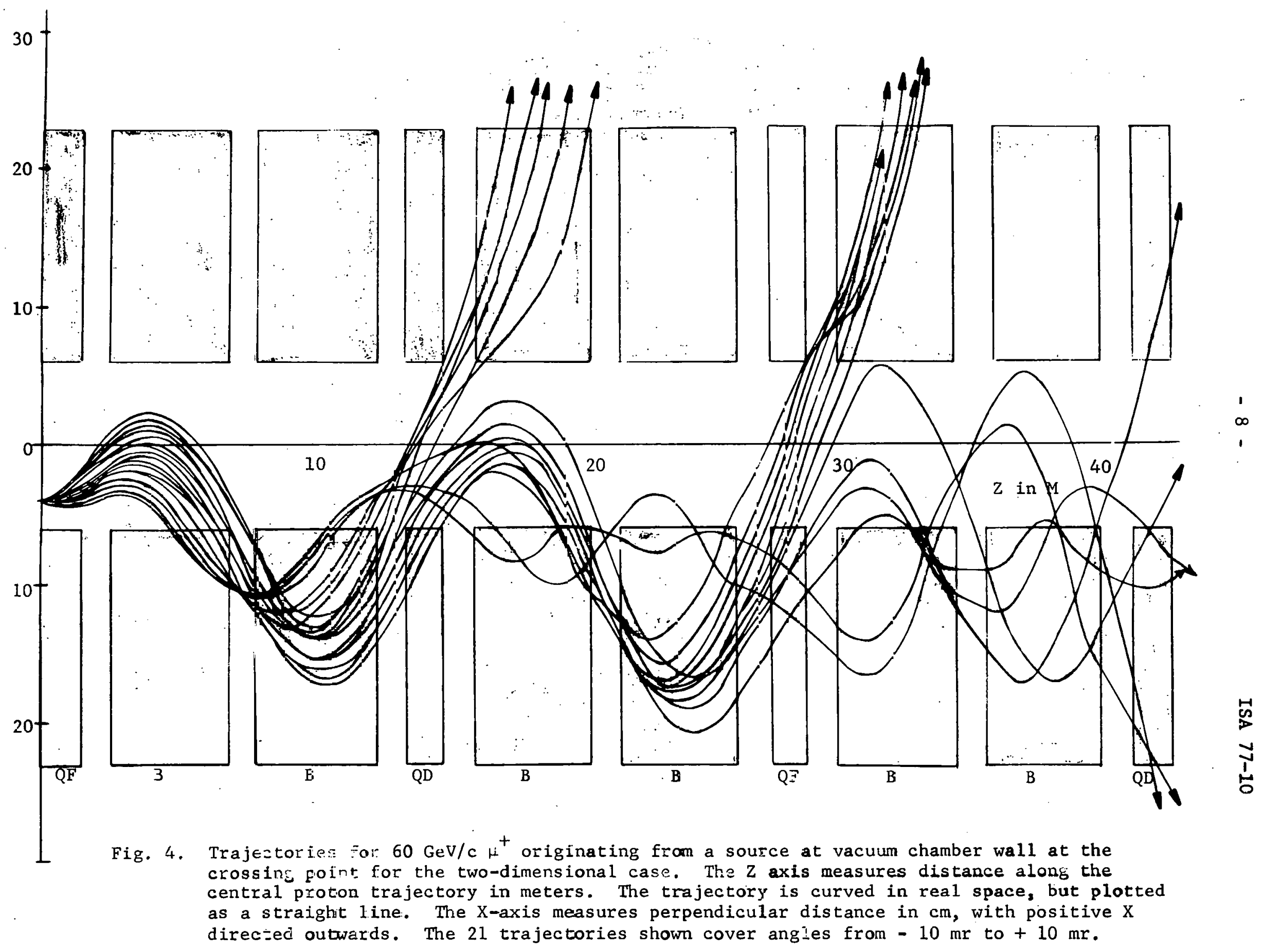


From these three qualitative conclusions can be drawn, which seem likely to be valid in the real, three-dimensional case also:

1) Trapping is energy-dependent, being a large effect for muons of $100 \mathrm{GeV}$ or more, and small below $50 \mathrm{GeV}$.

2) More $\mu^{+}$emerge on the outer side, although the dipole field deflects them to the inside.

3) The dipole downstream from a quadrupole seems to be the favorite spot for muons to emerge.

In Fig. 5 we show the trajectories from a scraper position located at the upstream end of the straight section. Since only quadrupoles are involved the trajectories are much like those for a source at the crossing point, but somewhat more strongly defocussing. There does not seem to be any appreciable trapping effect for this geometry. Of course, the scraper would not be located exactly on-axis, but trajectories for off-axis positions are very similar to those in Fig. 5 .

\section{Illustrative Three-Dimensiona1 Trajectories}

We have used the CERN program, HALO, to simulate muon behavior in a more realistic three-dimensional case. HALO is a Monte Carlo program which generates parent pions or kaons, and their decay muons. The charged particles are tracked through magnetic fields using a field map for points outside the central aperture. Energy loss and scattering are introduced according to the material through which the muons pass. Muon fluxes predicted by HALO have been satisfactorily compared with experimental measurements at NAL ${ }^{6}$.

The main purpose of HALO is to generate distributions of muon position, angle, energy, etc., which can be output as histograms. There is also an option to output individual trajectories in three dimensions. In this section we present a few trajectories, while section 5 will give some muon distributions. These HALO runs are for magnet dimensions and positions and field maps that are approximately those of the ISABELLE proposal; but they do not correspond exactly, so the results are illustrative ones only.

6. Craig D. Moore, "Comparison of HALO Predictions with Experimenta1 Measurements of offsite Muons Arising from $275 \mathrm{GeV} / \mathrm{c}$ Muon Line Operations", FNAL TM - 680 (Aug. 10; 1976). 


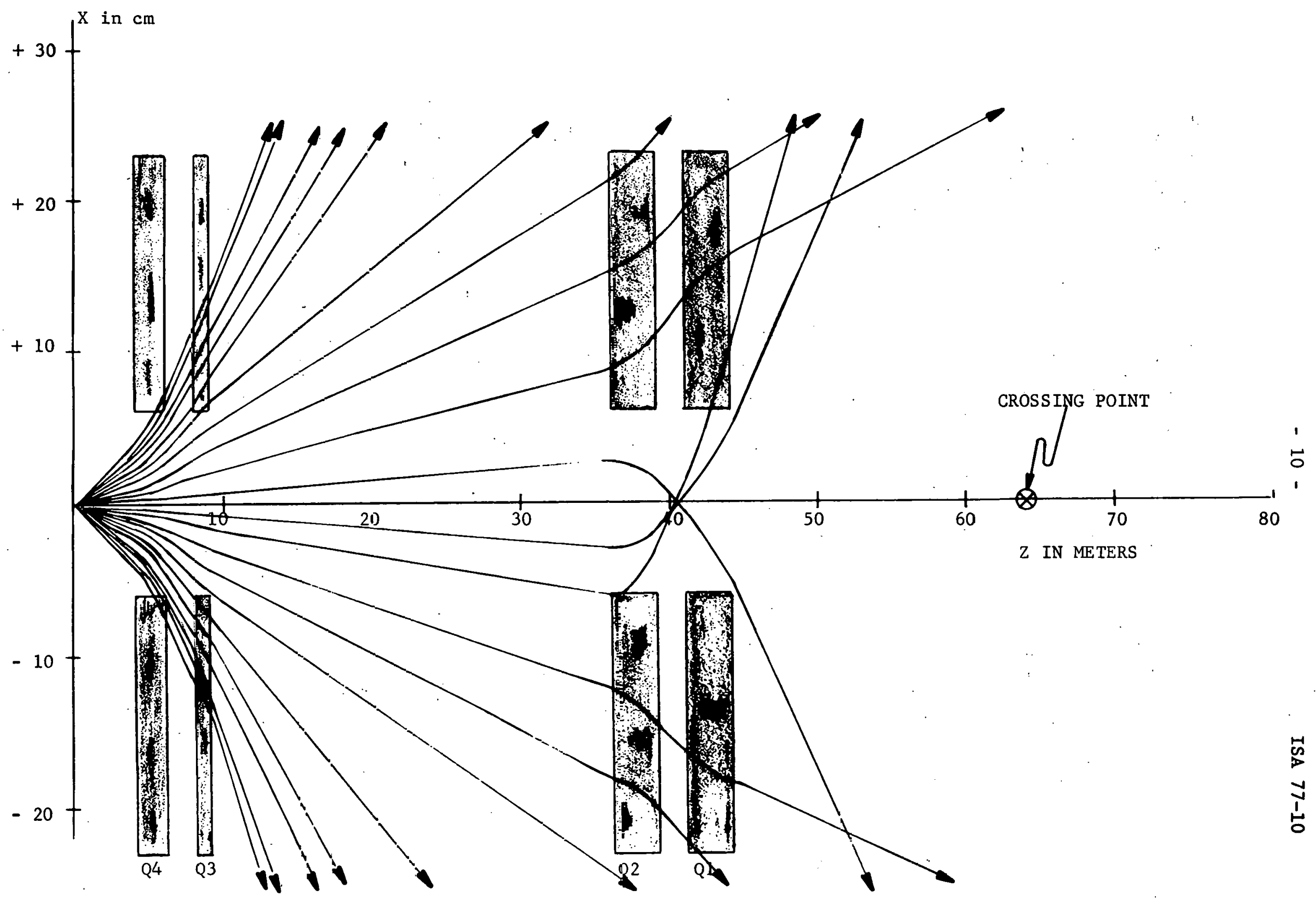

Fig. 5. Trajectories or $60 \mathrm{GeV} / \mathrm{c}^{+}$o 0 ininating from a source at scraper fosition centered in the vacuum tank for the two-dimensional casi. These trajectories are for angles from $-10 \mathrm{mr}$ to +1 and $-\frac{1}{2}$. to $+9 \frac{1}{2}$ so those above this axis inierpolate between those belcw. 
In general the HALO trajectories substantiate the conclusions drawn from the two-dimensional orbits. There is more information' in them, and it is hard to display their three-dimensional features in two-dimensional plots. Consequently we include only a small sample of trajectories with source in the lattice quadrupole in Figs. 6-8, selecting muons with initial momentum above $30 \mathrm{GeV} / \mathrm{c}$ since lower momenta are of less interest.

The typical pattern in these cases is for a single half-oscillation in $\mathrm{x}$ with the muon leaving to the outside, and relatively small motion in y. In example 非1, however, which is the highest momentum in a sample of 100 trajectories, the motion in $x$ starts out as a series of oscillations about the stable orbit, but the y motion passes out of the magnet structure about 30 meters from the source.

\section{Illustrative Distributions}

HALO will also provide histograms to show muon distributions at various points. With the source at the intersection point with 150,000 pions generated there are 2374 muons generated before the pions hit solid material in which the program considers them to be obsorbed. Figure' 9 shows the muon distributions 122 meters downsteam. At this point most muons are in the sand just outside the tunnel, which was given a radius of $210 \mathrm{~cm}$ for the program.

Overall the muons are concentrated in a broad beam about $400 \mathrm{~cm}$ wide, emerging tangentially. The dip in the $y$-distribution near $y=0$ is believed to be due to scattering and absorption by the ring magnets of muons emitted at small angles to the inside, which are intercepted by the ring as it curves inwards.

With the source in the lattice quadrupole fewer pions decay because they tend to hit solid material sooner. A population of 150,000 pions yielded 474 muons. Figure 10 shows the muon distribution 51 meters downstream. The most obvious interesting feature is the division of the $x$ distributions into a sharp outside peak and a broader one still close to the magnet ring. This peak is believed to consist of trajectories such as those shown in Figs. 4, 6, and 7, that are lost following the next down- 


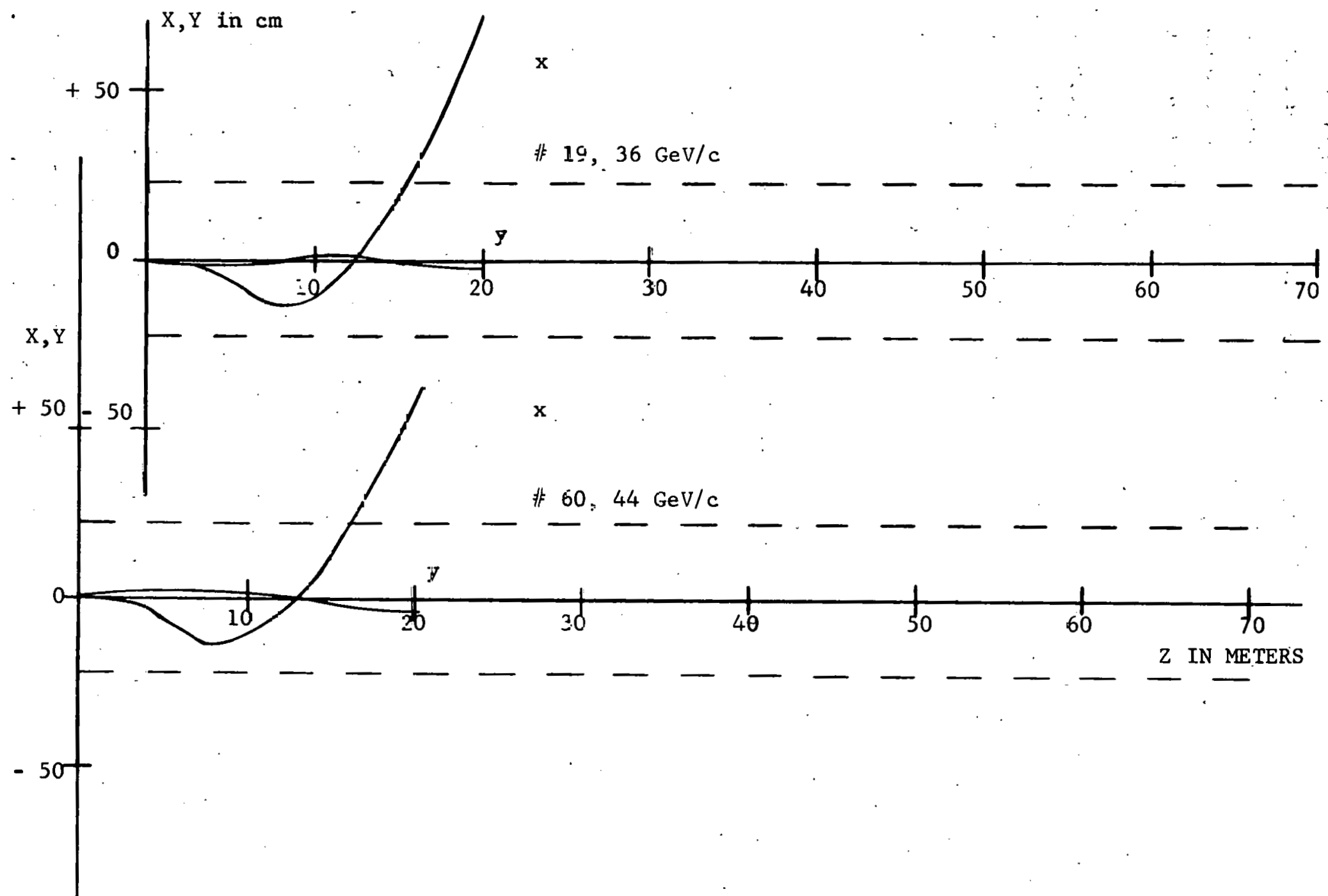

Fig. 6. Twc trajectories generated by HhLO with $\mathrm{x}$ and $\mathrm{y}$ motion shown, for source at center of vacuum in a latice quadrupole. Dotted lines indicate the extent of magnet core steel approximately, without showing individual dipole and quadrupole magnets. Their arrangement is shown in Fig. 4. 


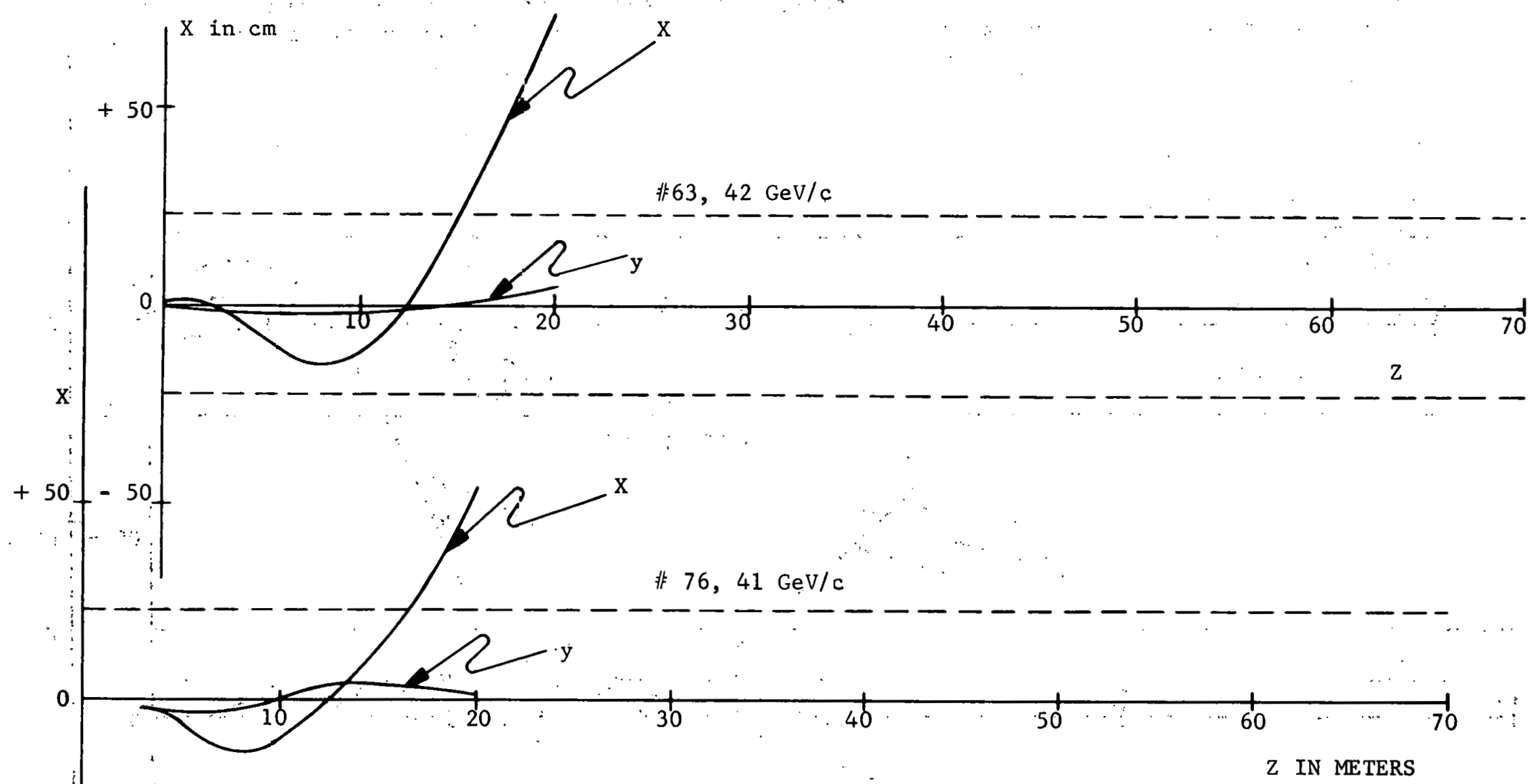

Fig. 7. Two trajectories generated by HALO with $\mathrm{x}$ and $\mathrm{y}$ motion shown, for source at center of vacium in a lattice quadrupole. Datted lines indicate the extent of magnet core steel approximately, without showing individual dipole and quadrupole magnets. Their arrangement is shown in Fig. 4. 


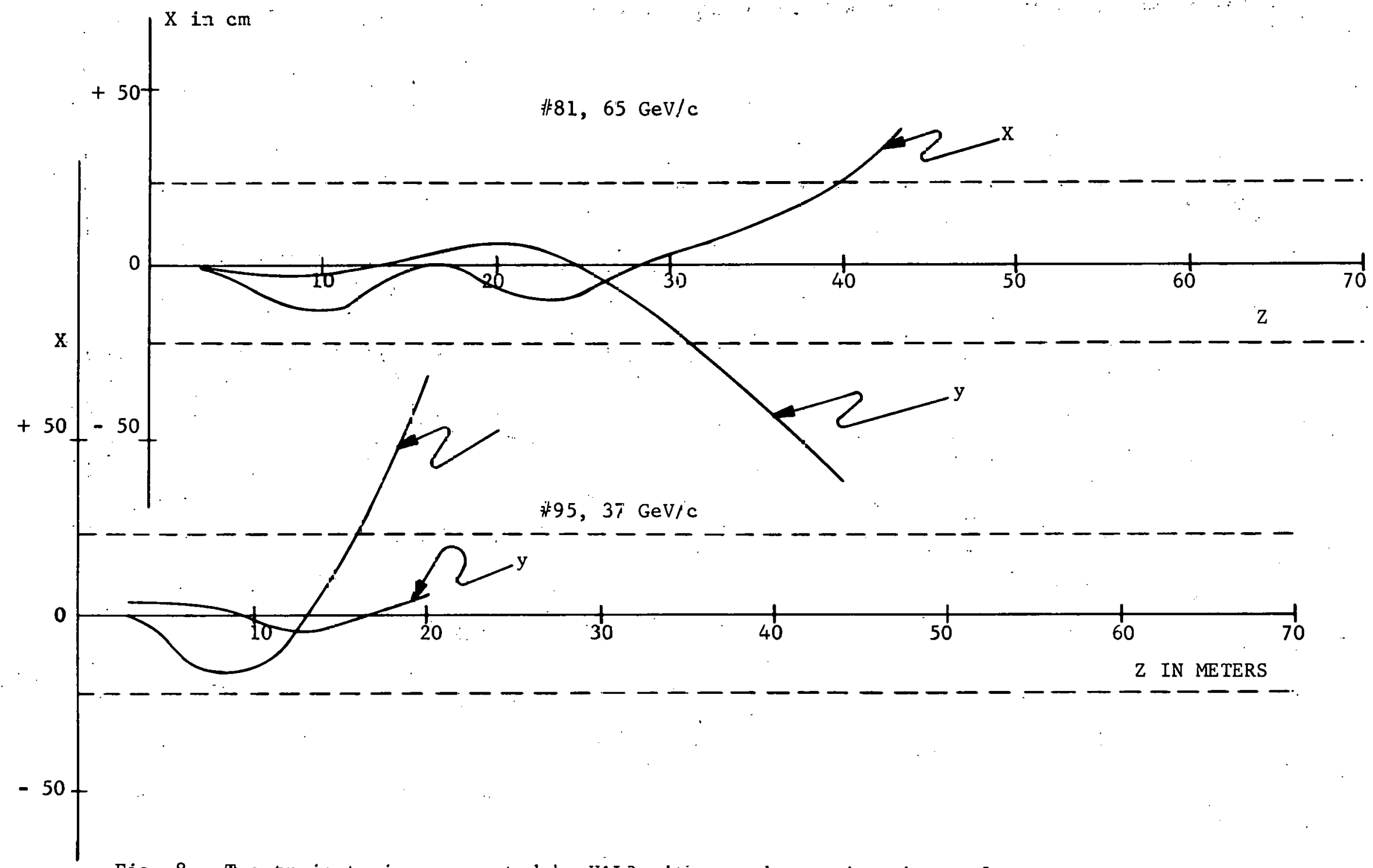

Fig. 8. Two trajectcries generated by HiLO witi $x$ and $y$ motion shown, for source at center of vacuum in a lattice quadrupole. Dotted lines indicate the extent of magnet core steel approximately, without showing individıal dipole and quadrupole magnets. Their arrangement is siown in $\mathrm{Fig} .4$. 


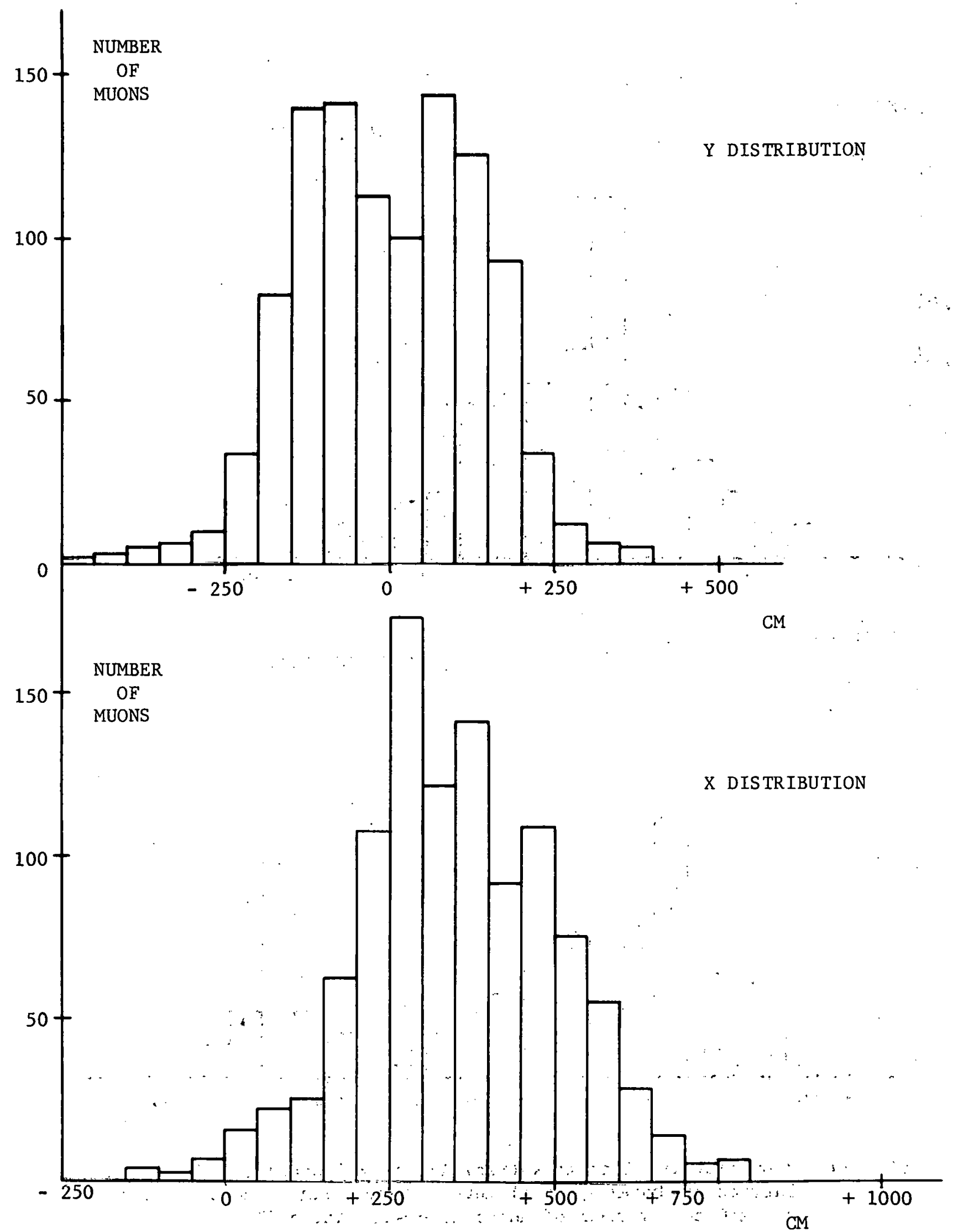

Fig. 9. Muon distributions in $x$ (radial) and $y$ (vertical) for source at the crossing point, and 122 meters downstream. Tunnel wall is at $\mathrm{X} \approx 210 \mathrm{~cm}$. Number of muons in distribution 1059. 


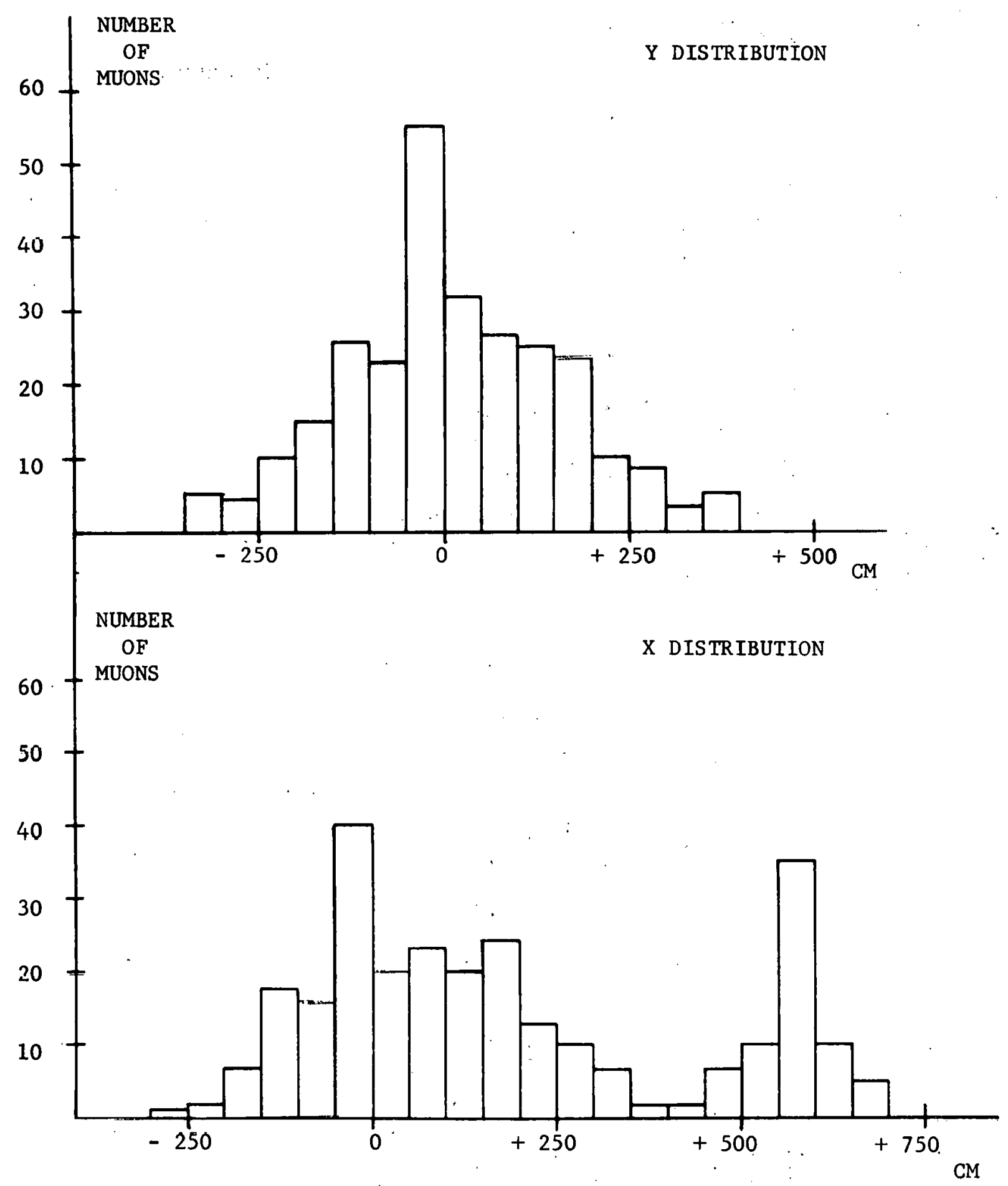

Fig. 10. Muon distributions in $x$ (radial) and $y$ (vertical) for source at lattice quadrupole, at 51 meters downstream. Tunnel wall is at $x \approx 210 \mathrm{~cm}$. Number of muons in distribution 270 . 
stream quadrupole. The two-dimensional $\mathrm{x}, \mathrm{y}$ histograms also show an interesting structure in which this peak is the outstanding feature, but more detailed study would probably reveal other interesting structure.

Finally, we show distributions for the source located at the scraper location in Fig. 11. The distributions located 207 meters downstream look similar to those for the source at the crossing point.

Using HALO to obtain quantitative estimates of muon fluxes that may be useful for estimating shielding requirements and for studying backgrounds in experimental areas is still in a preliminary stage. Obtaining results for cases where the protons interact at regions within the normal lattice may be particularly difficult since (1) the complicated nature of the orbits may indicate great sensitivity to the exact position of the interacting protons and (2) the program does not simulate direct muon production which becomes increasingly important in this region.

It is expected, however, that the most serious shielding problems will result from protons interacting in the experimental insertion regions because of the long path lengths available for hadron decay. It is therefore instructive to calculate the muon flux (available from two-dimensional histograms in HALO) at the position of the edge of the currently proposed muon shield resulting from protons which interact at the crossing regions.

The result of such a calculation gives $2 \times 10^{-9}$ muons per $\mathrm{cm}^{2}$ per proton. The most stringent requirement for muon flux is that of the site boundary (see Ref. 1) which is $\sim 15 \times 10^{4} \mathrm{\mu} / \mathrm{cm}^{2}$ year. Using a $1 / \mathrm{r}^{2}$ falloff from the shield edge to the site boundary, a $0.1 \%$ loss of the protons at the crossing region, and a total proton fill as detailed in Ref. 1, the HALO prediction becomes $4.5 \times 10^{4} \mathrm{~m} / \mathrm{cm}^{2}$, year, we 11 within the required 1 imit. 

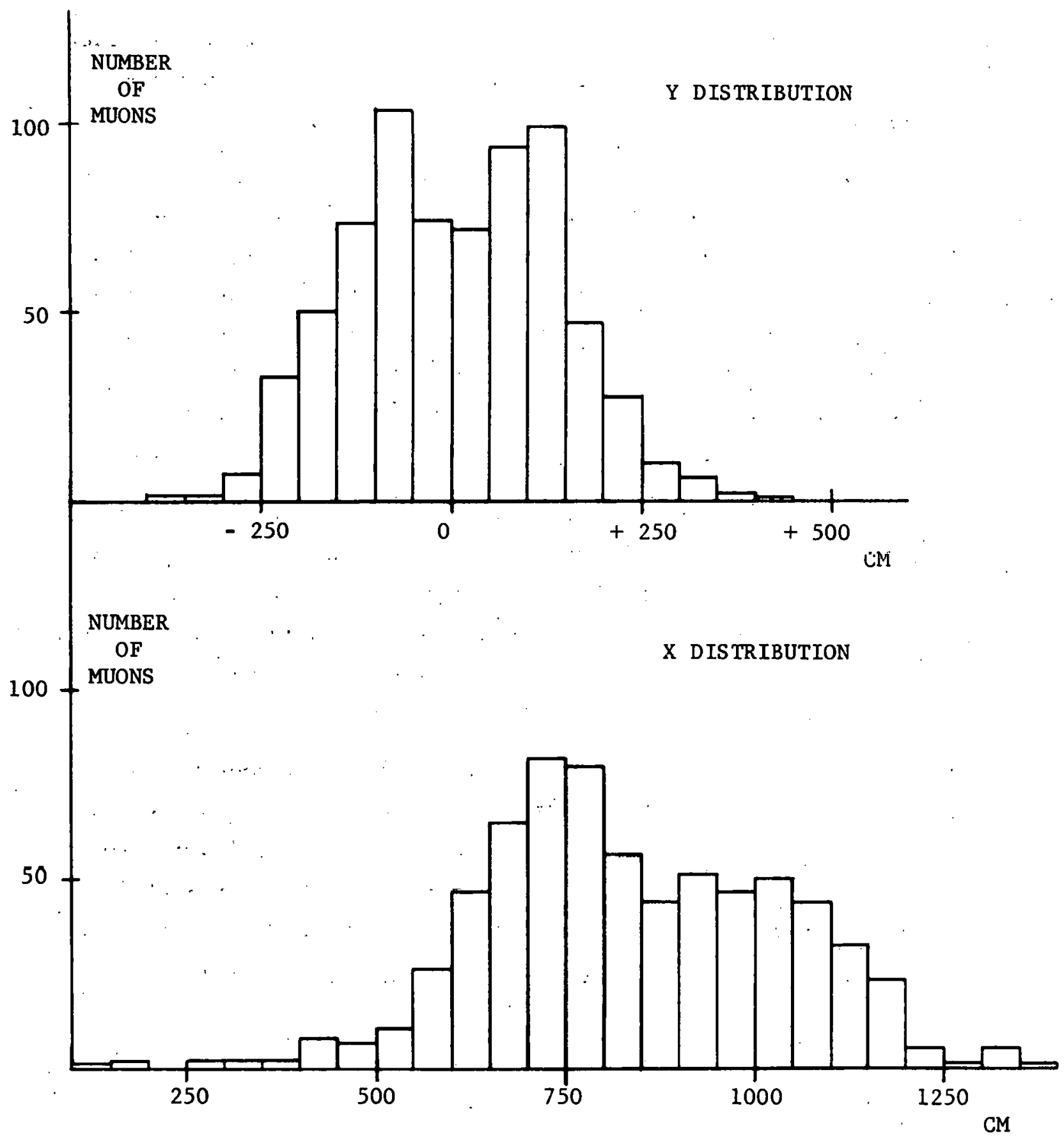

Fig. 11. Muon distributions in $x$ (radial) and $y$ (vertical) for source at scraper, at 207 meters downstream. Tunnel wall is at $x \approx 210 \mathrm{~cm}$. Number of muons in distribution 703. 
6. Qualitative Conclusions

As stated above, the quantitative use of HALO is in a preliminary stage. Qualitatively, however, the following conclusions may be drawn:

a. Magnetic field effects are important for an understanding of muon trajectories and fluxes.

b. Quadrupoles in the ISABELLE insertions do not generate trapped orbits.

c. Dipoles in the lattice produce a significant trapping effect.

d. This effect is selective and primarily affects muons of high momentum and small angle, which are not numerous.

e. Trapping therefore will not reduce the muon shielding requirements by a large factor.

f. HALO appears to be a suitable program for studying specific details as they may affect shielding needs and backgrounds in experimental areas.

Distr.: External 